\title{
Prenatal care perception of Saudi females of childbearing age: an epidemiological study
}

\author{
Saleh F. Alqifari ${ }^{1 *}$, Fahad A. Alharbi ${ }^{2}$, Abdulrahman Algeffari ${ }^{3}$
}

${ }^{1}$ College of Pharmacy, University of Arizona, Tucson, Arizona, United States

${ }^{2}$ College of Pharmacy, Ohio Northern University, Ada, Ohio, United States

${ }^{3}$ College of Medicine, Qassim University, Saudi Arabia

Received: 14 February 2019

Accepted: 07 March 2019

\section{*Correspondence:}

Dr. Saleh F. Alqifari,

E-mail: alqifari@pharmacy.arizona.edu

Copyright: ( ) the author(s), publisher and licensee Medip Academy. This is an open-access article distributed under the terms of the Creative Commons Attribution Non-Commercial License, which permits unrestricted non-commercial use, distribution, and reproduction in any medium, provided the original work is properly cited.

\section{ABSTRACT}

Background: The purpose of this study is to assess perception of Saudi women of childbearing age regarding prenatal care.

Methods: Participants were invited to participate in a voluntary online survey. The brief survey consisted of 15 basic multiple-choice questions administered in Arabic and delivered as a secure link through private social media messaging services. The survey permitted only 1 attempt per user. In order to reach and capture non-respondents, 2 follow-up reminders 10 days apart were sent through the same channels of communication. The survey data was collected and analyzed using the survey software platform, Qualtrics by Qualtrics (C LLC 2017.

Results: Approximately 9\% of participants reported previous pregnancy without use of prenatal care. Nearly $12 \%$ of respondents thought that prenatal care is only indicated for malnourished pregnant females, and $7 \%$ reported not knowing who prenatal care is specifically indicated for. Nearly $21 \%$ reported prenatal care should start after confirmation of pregnancy, $14 \%$ thought proper start is anytime within the first 3 months, $8 \%$ reported proper start should be after confirmation of healthy pregnancy and $8 \%$ reported not sure when to initiate prenatal care. Approximately $12 \%$ thought taking prenatal vitamins should be once weekly or some days of the week, while $5 \%$ do not advice women to take prenatal vitamins during pregnancy at all.

Conclusions: The majority of Saudi women are well educated on prenatal care. However, women in early and late twenties are less aware of proper usage of prenatal care.

Keywords: Malnourished pregnant females, Prenatal, Vitamins, Women

\section{INTRODUCTION}

Prenatal care is an essential stage towards a healthy pregnancy and a child later in life. ${ }^{1}$ It is categorized as a preventive healthcare with the primary aim of preventing potential health problems for both the mother and the developing fetus. ${ }^{2}$ It promotes a healthy lifestyle among pregnant mothers and children. ${ }^{1,3}$ Adequate nutrition intake before and during pregnancy was found to be important for fetal and maternal health. ${ }^{1}$

Therefore, maternal diet is important to cover the body nutritional requirements for a healthy fetus development and maternal and infant health. ${ }^{4}$ Thus, prenatal nutritional supplementation of iron and folic acid or iron-folic acid (IFA) are often prescribed for healthier infants. ${ }^{5}$ 
Many studies indicated the positive effect of these supplementations on birth weight which may become associated with health, education, and income in infants' future life. ${ }^{6-8}$ In 2016, the WHO reported 2.6 million infant deaths during their first month, making children mortality an important public health issue in the entire world. ${ }^{9,10}$ The vast majority of this mortality rate takes place in developing countries. ${ }^{10}$ In Saudi Arabia, infant mortality rate is 7.5 infant deaths per 1,000 live births and $8.4 \%$ of the entire births were born with a low birth weight in 2014. ${ }^{11}$ These high numbers raise many questions regarding the prenatal care perception of women in childbearing age in Saudi Arabia. To our knowledge, no attempt had been made in Saudi Arabia to assess prenatal care perception in childbearing age women.

The purpose of this study is to assess the perception of women of childbearing age in Saudi Arabia in regard to prenatal care. The study aims to assess the knowledge and perception of Saudi mothers about prenatal care.

\section{METHODS}

In 2016, Saudi Arabian women of childbearing age and with a mean age of 30 years were invited to participate in a voluntary online survey (Table 1). The brief survey consisted of 15 basic multiple-choice questions delivered as a secure link through private social media messaging services. 1855 Saudi women completed the survey. The primary questions consisted of age of the participant, their academic level, number of pregnancies, number of births, number of abortions, whether mothers have used supplements during any of their pregnancies, if they believe prenatal vitamins are just for malnourished mothers, the period that they believe that prenatal supplements should be used, their choice of prenatal supplements, and lastly if they would recommend dietary supplements with every pregnancy. The survey permitted only one attempt per user so that it would reduce redundancy and improve accuracy of data. To reach out and capture non-respondents, 2 follow-up reminders 10 days apart were sent through the same channels of communication. The survey included a general message to mothers inviting them to take part in the survey. The study protocol was granted Institutional Review Board "IRB" approval from Ohio Northern University located in Ada, Ohio United States. The survey data was collected and analyzed using the online survey software platform, Qualtrics.

Table 1: Characteristic of women participated in the online survey.

\begin{tabular}{|l|l|}
\hline Characteristic & $18-40$ years \\
\hline Age range & 30 years \\
\hline Mean age & 61 \\
\hline Educational level & 303 \\
\hline Less than high school & 1385 \\
\hline $\begin{array}{l}\text { High school diploma } \\
\text { Bachelor's degree }\end{array}$ & 106 \\
\hline Postgraduate degree & 2.3 \\
\hline $\begin{array}{l}\text { Average number of pregnancies per } \\
\text { woman }\end{array}$ & 0.3 \\
\hline $\begin{array}{l}\text { Average number of abortions per } \\
\text { woman }\end{array}$ & \\
\hline
\end{tabular}

\section{RESULTS}

Approximately $6 \%$ of participants reported they had previous pregnancy(s) without the use of prenatal care. The results noted about $90 \%$ of women have used prenatal care during previous pregnancies or abortions. Only $8 \%$ reported pregnancy without the use of prenatal care, most of which were mainly below the age of 30 years. Nearly $14 \%$ indicated that prenatal care is only indicated for malnourished females, and $8 \%$ reported not knowing who prenatal care is specifically indicated for (Figure 1).

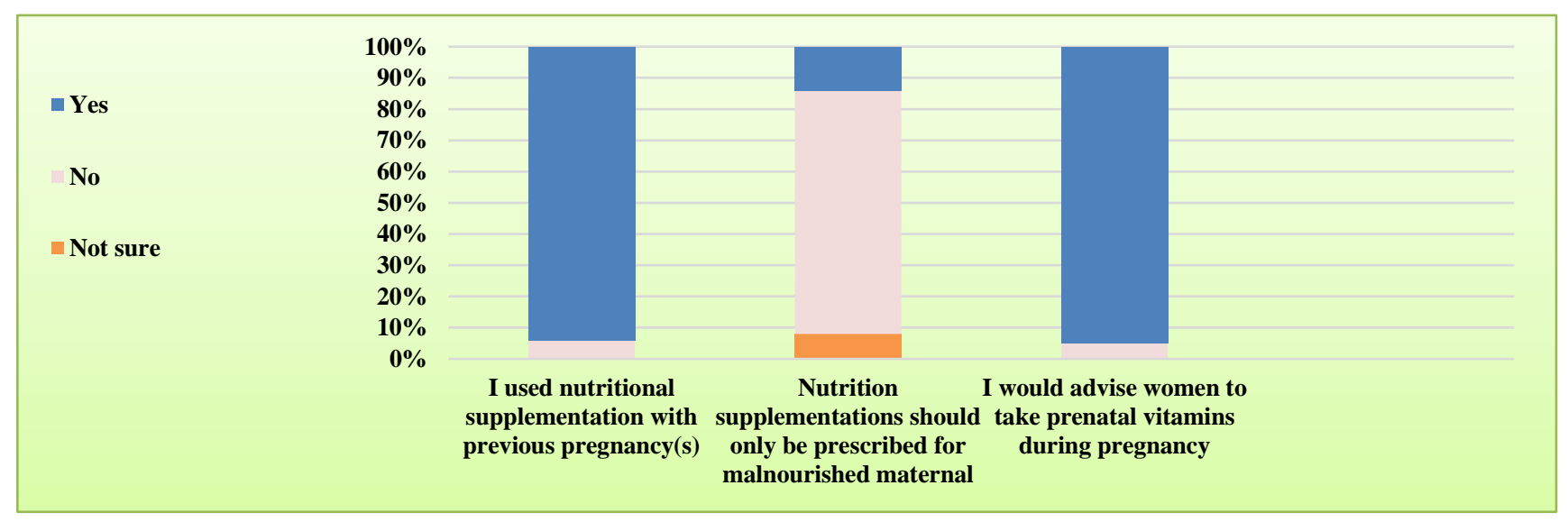

Figure 1: Assessment of the previous usage of nutrition supplementation and if participants think it is only indicated for malnourished maternal; also, if participants would advise other women to take nutrition supplementation. 
The $14 \%$ of women who thought that prenatal care was just for malnourished females also formed $90 \%$ of the $8 \%$ of the women who undergone pregnancies without prenatal care. Approximately $12 \%$ thought taking prenatal vitamins should be weekly or some days of the week, while $5 \%$ do not advise women to take prenatal vitamins during pregnancy (Figures 1 and 2). In Figure 2, $14 \%$ of the participants used nutrition supplementation weekly when the rest $86 \%$ used them daily during their previous pregnancy(s).

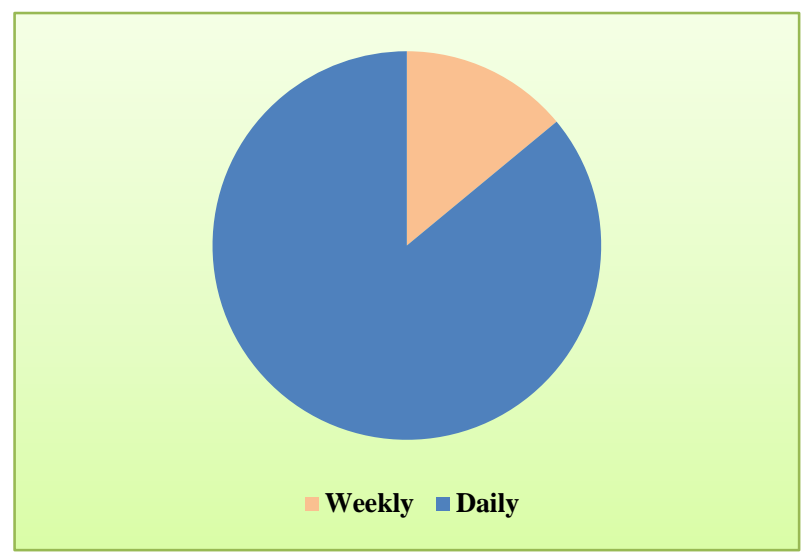

Figure 2: How often nutrition supplementation should be used?

Mothers who had 2 pregnancies reported that they were well informed about prenatal care and had used prenatal care in the past. Mothers who had 3 pregnancies, $97 \%$ of them recommended the use of prenatal care for all women of childbearing age. Nearly $21 \%$ reported that prenatal care should start after confirmation of pregnancy, $8 \%$ reported proper start should be after confirmation of a healthy fetus, $14 \%$ thought proper start is any time within the first 3 months, and $8 \%$ reported not sure when to initiate prenatal care (Figure 3). Authors calculated that about $90 \%$ of women who are informed about prenatal care lack knowledge of when care should be initiated. Authors also noted that the most recognized supplements by Saudi women are folic acid, calcium, iron, and daily multivitamins.

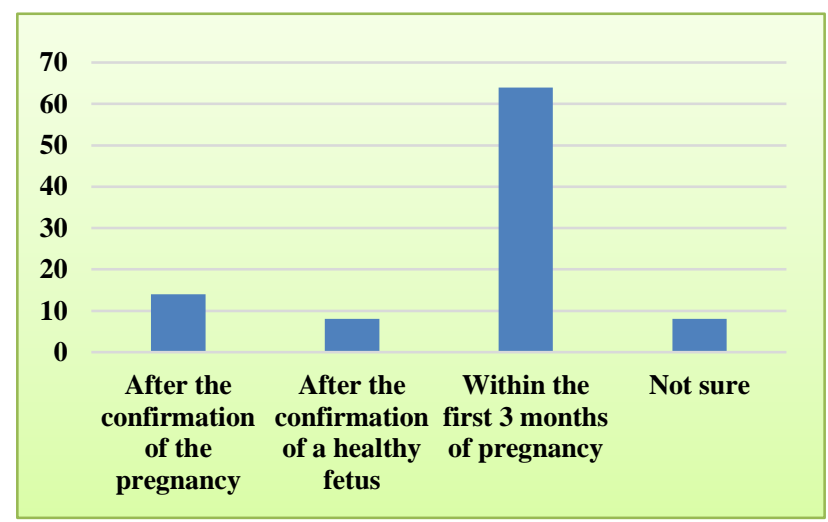

Figure 3: When nutrition supplementation should be started?
In Figure 3, 14\% of the survey participants answered that nutrition supplementation should be taken after the confirmation of the pregnancy and $8 \%$ thought it was should be taken after the confirmation of a healthy fetus. However, $64 \%$ thought that nutrition supplementation should be taken within the first 3 months and $8 \%$ were not sure of when nutrition supplementation should be taken.

\section{DISCUSSION}

This study suggests that most women who had received prenatal care were above the age of 30 years showing that extent of knowledge of prenatal care increased proportionally with age. This finding is interesting as it negates the study findings published by Saudi Medical Journal in 2013 and conducted in healthcare centers during antenatal visits showed that there was a negative linear relationship between health education score of women and their age. ${ }^{12}$

A closer look at the data shows that women knowledge of prenatal care came from previous experiences as $97 \%$ of mothers who had 3 pregnancies recommend the use of prenatal care. This is consistent with the finding of AlShammari et al, who shows that access to care was a barrier and women had to learn to take prenatal care from the previous experiences. ${ }^{13}$

The result of the question asking about when to use prenatal supplement shows that more than $90 \%$ of women who are informed about prenatal care do not know when it should be initiated. This indicates that there is an educational gap that needs to be addressed. Authors believe that need for educational efforts to be carried out across Saudi Arabia to educate mothers and young females in schools and public events is pressing. Although educational efforts need to target general awareness about the value of prenatal care, it seems that large percentage of Saudi woman have good basic knowledge but lack awareness about details related to prenatal care such as correct time of initiation of prenatal care.

\section{CONCLUSION}

In conclusion, authors noted that women in their early and late twenties are not well informed about prenatal care. This indicates that there is need for educational efforts to be carried out across Saudi Arabia. Moreover, women need to be educated that prenatal care should be utilized in every pregnancy despite the age of the mother. Further research is needed to learn more about the impact of the lack of prenatal care awareness on areas such as birth defects and miscarriages.

Funding: No funding sources Conflict of interest: None declared

Ethical approval: The study was approved by the Institutional Ethics Committee 


\section{REFERENCES}

1. Emmett PM, Jones LR, Golding J. Pregnancy diet and associated outcomes in the Avon Longitudinal Study of Parents and Children. Nutr Rev. 2015;73(Suppl 3):154-74.

2. Hambidge KM, Krebs NF. Strategies for optimizing maternal nutrition to promote infant development. Reprod Health. 2018;15(Suppl 1):87.

3. Black RE, Allen LH, Bhutta ZA, Caulfield LE, de Onis M, Ezzati M, et al. Maternal and child undernutrition: global and regional exposures and health consequences. Lancet. 2008;371(9608):24360 .

4. Huijgen NA, van de Kamp ME, Twigt JM, de Vries JH, Eilers PH, Steegers EA, et al. The preconception dietary risk score; a simple tool to assess an inadequate habitual diet for clinical practice. e-SPEN J. 2014;9(1):e13-9.

5. Li C, Zeng L, Wang D, Yang W, Dang S, Zhou J, et al. Prenatal micronutrient supplementation is not associated with intellectual development of young school-aged children. J Nutr. 2015;145:1844-9.

6. Tette EMA, Sifah EK, Nartey ET, Nuro-Ameyaw P, Tete-Donkor P, Biritwum RB. Maternal profiles and social determinants of malnutrition and the MDGs: What have we learnt? BMC Public Health. 2016;16:214.

7. Langley-Evans SC. Nutrition in early life and the programming of adult disease: a review. J Hum Nutr Diet. 2015;28:1-4.
8. Northstone K, Emmett P, Rogers I. Dietary patterns in pregnancy and associations with sociodemographic and lifestyle factors. Eur J Clin Nutr. 2007;62(4):471-9.

9. Abir T, Ogbo F, Stevens G, Page A, Milton A, Agho $\mathrm{K}$. The impact of antenatal care, iron-folic acid supplementation and tetanus toxoid vaccination during on child mortality in Bangladesh. Plos ONE. 2017;12(11):1-14.

10. World Health Organization. Newborns: reducing mortality. Fact Sheet. Available at: http://www.who.int/news-room/factsheets/detail/newborns-reducing-mortality.

11. Ministry of Health (Saudi Arabia). Saudi Arabia Health Statistical Yearbook 2014.

12. Al-Ateeq MA, Al-Rusaiess AA, AlDughaither AA. Perceptions and effects of antenatal education. Saudi Med J. 2013;34(12):1287-93.

13. Al-Shammari SA, Khoja T, Jarallah JS. The pattern of antenatal visits with emphasis on gestational age at booking in Riyadh Health Centres. J Royal Soc Health. 1994;114(2):62-6.

Cite this article as: Alqifari SF, Alharbi FA, Algeffari A. Prenatal care perception of Saudi females of childbearing age: an epidemiological study. Int J Reprod Contracept Obstet Gynecol 2019;8:1246-9. 